\title{
GeneXpert positivity and evaluation of rifampicin resistance among endometrial biopsy specimens for the diagnosis of Genital Tuberculosis
}

\author{
Bineeta Kashyap, Dibyashree, Rituparna Saha, NP Singh, Puneeta Hyanki, \\ Ashwani Khanna \\ Correspondence: Dr Bineeta Kashyap: Associate Professor, Department of Microbiology, \\ University College of Medical Sciences and Guru Teg Bahadur Hospital, New Delhi. Email - \\ dr_bineetakashyap@yahoo.co.in
}

Distributed under Attribution-NonCommercial - Share Alike 4.0 International (CC BY-NC-SA 4.0)

\begin{abstract}
Objective: The aim of this study was to find out the GeneXpert positivity among endometrial biopsy specimens for diagnosis of genital tuberculosis. Materials and Methods: A retrospective study was conducted from January 2019 to June 2019 on all women clinically and radiologically diagnosed with infertility, dyspareunia, menstrual irregularities and pelvic inflammatory disease. Endometrial biopsies were taken from all cases and subjected to GeneXpert test for detection of Mycobacterium tuberculosis. Result: Total number of suspected cases was 62 with age ranging from 19 years to 65 years. Majority of suspected cases were from the age group 21-30 years (54.8\%). Five out of 62 (8\%) cases tested positive for Mycobacterium tuberculosis by GeneXpert which were all sensitive to Rifampicin. Among these 5 cases, 2 were reported as medium positive and 3 as very low positive based on cycle threshold value. Conclusion: GeneXpert on endometrial biopsy may prove to be a reliable diagnostic modality for genital tuberculosis as well as multidrug resistance tuberculosis in females leading to timely management of the disease.
\end{abstract}

Keywords: Gene, biopsy, tuberculosis.

Genital tuberculosis is a chronic disease in females with low grade symptoms. It can result in infertility, dyspareunia, menstrual irregularities and chronic pelvic inflammatory disease ${ }^{1}$. Fallopian tube in females are most frequently affected $(90-100 \%)$ followed by endometrium (50-60), ovaries (20-30), cervix (5-15\%), vulva and vagina $(1 \%)^{2}$. Infertility is a worldwide health problem, genital tuberculosis being one of its main causes accounting for nearly $18 \%$ infertility cases in India ${ }^{3}$.
Therefore, country like India having the highest incidence of tuberculosis in the world needs early and aggressive strategies to diagnose and treat tuberculosis. Diagnosis of female genital tuberculosis by microscopy and culture is difficult due to its paucibacillary nature ${ }^{4}$. Histopathological diagnosis demonstrates typical caseous granulomatous lesions with giant epitheloid cells suggestive of tuberculosis but not diagnostic as these also appear in fungal infections and sarcoidosis ${ }^{5}$. In December

Received: $16^{\text {th }}$ September 2019. Accepted: $19^{\text {th }}$ November 2019.

Kashyap B, Dibyashree, Saha R, Singh NP, Hyanki P, Khanna A. GeneXpert positivity and evaluation of rifampicin resistance among endometrial biopsy specimens for the diagnosis of Genital Tuberculosis. The New Indian Journal of OBGYN. 2020; 6(2): 119-22. 
2010, GeneXpert was endorsed by World Health Organisation for the diagnosis of pulmonary and extrapulmonary tuberculosis ${ }^{6}$.

GeneXpert is an automated nucleic acid amplification test. It has disposable single use multi-chambered plastic cartridge preloaded with liquid buffers and lyophilised reagent beads required for sample processing, DNA extraction and heminested real time polymerase chain reaction (PCR) ${ }^{7}$. It simultaneously detects the presence of Mycobacterium tuberculosis and rifampicin resistance by amplifying a sequence of $r p o \mathrm{~B}$ gene specific to members of Mycobacterium tuberculosis complex and probe for mutations within the 81-bp rifampicin resistancedetermining region (RRDR) of the $r p o \mathrm{~B}$ gene which is detected by five overlapping molecular beacons ${ }^{8}$. Although several studies have evaluated the role of GeneXpert in diagnosis of tuberculosis in extrapulmonary samples including tissue and body fluids but its role in diagnosing genital tuberculosis is still very limited. Not many studies assessing the utility of GeneXpert for the diagnosis of genital tuberculosis has been found. The aim of this study was to find out the GeneXpert positivity among endometrial biopsy specimens for diagnosis of genital tuberculosis in females in a tertiary care hospital.

\section{Materials and Methods}

A retrospective study was conducted from January 2019 to June 2019 on all women clinically and radiologically diagnosed with infertility, dyspareunia, menstrual irregularities and pelvic inflammatory disease at GTB hospital, New Delhi. Endometrial biopsies were taken from all cases and subjected to GeneXpert test for detection of Mycobacterium tuberculosis at DOTS centre of GTB Hospital, New Delhi. Collected data were expressed in tables with number and percentage.

\section{Results}

Total number of suspected cases was 62 with age ranging from 19 years to 65 years. Majority of suspected cases were from the age group 21-30 years (54.8\%) as shown in figure 1. Maximum number of cases presented in the month of January and June which accounted for $24 \%$ each (figure 2). Five out of $62(8 \%)$ cases tested positive for Mycobacterium tuberculosis by GeneXpert which were all sensitive to Rifampicin. Among these 5 cases, 2 were reported as medium positive and 3 as very low positive based on cycle threshold value (Table 1).
Table 1: Results of GeneXpert $(\mathrm{N}=62)$

\begin{tabular}{lll}
\hline GeneXpert test & & No (\%) \\
\hline Positive $(\mathrm{N}=5)$ & High & - \\
& Medium & $2(40 \%)$ \\
& Low & - \\
& Very low & $3(60 \%)$ \\
\hline Negative $(\mathrm{N}=57)$ & & \\
\hline
\end{tabular}

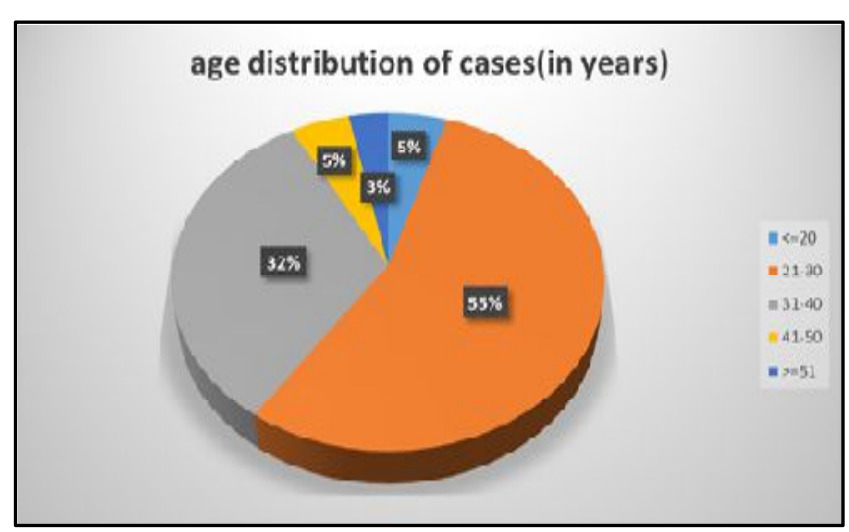

Figure 1: Percentage distribution of suspected cases in various age groups

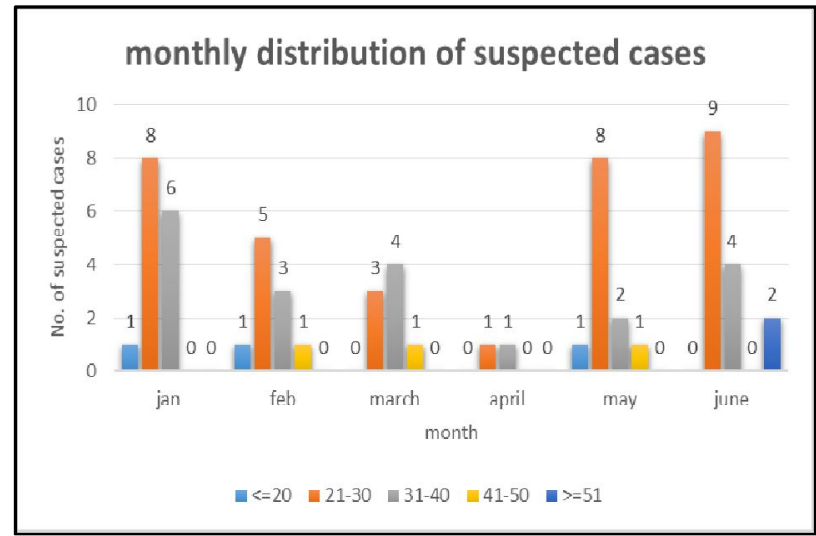

Figure 2: Distribution of suspected cases in various months

\section{Discussion}

Unlike pulmonary tuberculosis, it is difficult to clinically diagnose genital tuberculosis because majority of cases are asymptomatic or have non-specific symptomatology ${ }^{5}$. Lack of specific tests and varied clinical presentation of genital tuberculosis has led to underestimation of prevalence of genital tuberculosis ${ }^{9}$.

Very few studies have been done for GeneXpert as a diagnostic method of genital tuberculosis so not much 
data were available to compare our results. In our study GeneXpert positivity was $8 \%$ which is high compared to $2.9 \%$ positivity in the study conducted by Sharma et al in which they found out $100 \%$ sensitivity and specificity of GeneXpert for definite female genital tuberculosis confirmed by positive AFB culture, histopathology and diagnostic laparoscopy. However, the overall sensitivity was $46.6 \%$ taking into consideration the $3.3 \%$ cases having probable diagnosis of female genital tuberculosis by diagnostic laparoscopy which tested negative by GeneXpert as well as AFB culture and histopathology. The reason for the missed probable cases as quoted by the authors was paucibacillary nature of genital tuberculosis and certain limitations of GeneXpert itself which includes its sensitivity to high temperature and humidity which is prevalent in India. Blood contamination in endometrial biopsy can also render the GeneXpert negative. Despite these limitations, the authors have recommended GeneXpert as a useful adjunct in diagnosis of genital tuberculosis ${ }^{10}$.

GeneXpert scored negative in a study conducted by Garg et al in which diagnostic comparision of genital tuberculosis was done between GeneXpert and histopathological examination. In their study, among the 81 cases who presented with infertility 1 case $(1.23 \%)$ was found positive for tubercular endometritis and the rest 80 cases $(98.7 \%)$ had non tubercular findings suggestive of infertility. The study emphasized on sparse number of Mycobacterium tuberculosis in endometrial biopsy due to various reasons such as secondary nature of genital tuberculosis, limitation of infection to the fallopian tube and cyclical shedding of the endometrium making it evident that tuberculosis may not be detected in all cycles ${ }^{3}$. In another study conducted by Saxena et al, among 62 suspected cases of genital tuberculosis GeneXpert showed $1.6 \%$ positivity which was less compared to $22.9 \%$ positivity on culture. The study emphasized on both liquid and solid culture as an important role in diagnosing genital tuberculosis. They considered culture as gold standard for diagnosis which should be collaborated along with any rapid test for diagnosing tuberculosis ${ }^{11}$.

Farhana et al conducted a study in which 87 cases of suspected genital tuberculosis were subjected to Ziehl Neelson staining, culture on Lowenstein-Jensen media and GeneXpert assay. None of the samples were positive in $\mathrm{ZN}$ staining. However, $4.6 \%$ and $8.05 \%$ positivity was observed in culture and GeneXpert respectively. Two samples that were positive on culture were found negative on GeneXpert which could be because of blood contamination of endometrial biopsy sample as quoted by the authors. However, due to higher rate of detection and minimal technical expertise GeneXpert was recommended by the authors for rapid diagnosis and detection of drug resistant tuberculosis in genital tuberculosis ${ }^{4}$.

The XpertMTB/RIF (Xpert) assay detects resistance to Rifampicin which is also the surrogate marker for multidrug resistant (MDR) tuberculosis ${ }^{12}$. In our study all 5 positive cases by GeneXpert were Rifampicin sensitive excluding the possibility of MDR tuberculosis compared to the study by Saxena et al in which only one sample which tested positive by GeneXpert was rifampicin resistant ${ }^{11}$. In the study by Sharma et al all the 7 GeneXpert positive samples were rifampicin sensitive ${ }^{10}$.

The XpertMTB/RIF (Xpert) assay offers rapid TB diagnosis and quantitative estimation of bacterial burden through Cycle threshold $(\mathrm{Ct})$ values. $\mathrm{Ct}$ values inversely correlate with bacterial load i.e. lower $\mathrm{Ct}$ values represent a higher starting concentration of DNA template whereas higher $\mathrm{Ct}$ values represent a lower concentration of DNA template. The mean $\mathrm{Ct}$ values is categorized by the Xpertassay semi-quantitatively in relation to sample positivity as very low ( $>28$ cycles), low (23-28 cycles), medium(16-22 cycles) and high $(<16$ cycles $){ }^{13}$. In our study out of 5 positive samples by GeneXpert, 2 samples were medium positive and 3 samples were very low positive indicating paucibacillary nature of genital tuberculosis. None of the studies mentioned above has discussed about the cycle threshold values in their study so no data was available to compare our results. Proper utilization of ct values and its correlation with microscopy, culture and clinical profile can be useful for the clinician in appropriate treatment.

\section{Conclusion}

Significant morbidity and long term sequelae of genital tuberculosis with alarming increase in the prevalence of multidrug resistant tuberculosis has highlighted the urgent need of rapid diagnostic method which is accurate, feasible and affordable for use in resource limited setting. GeneXpert has evolved as an important breakthrough in the diagnosis of tuberculosis. 
The New Indian Journal of OBGYN. 2020 (January-June); 6(2)

The outcome of GeneXpert varies with sample type but it can be a promising diagnostic test on endometrial biopsy for diagnosing genital tuberculosis as well as MDR-TB in females leading to timely management of the disease. More studies on GeneXpert as a diagnostic modality in genital tuberculosis should be undertaken.

\section{Conflict of interest: None. Disclaimer: Nil.}

\section{References}

1. Grace GA, Devaleenal DB, Natrajan M. Genital tuberculosis in females.Indian J Med Res. 2019; 145(4): 425-36.

2. Schaefer G. Female genital tuberculosis.Clin Obstet Gyecol.1976;19(1):223-39.

3. Garg R, Agarwal N, Gupta M. Genexpert test and endometrial histological findings in infertile women. Int J Reprod Contracept Obstet Gynecol. 2018;7(4):1480-3.

4. Farhana A, Zahoor D, Manzoor M, Kanth F. Evaluation of Xpert MTB / RIF Assay for the Detection of Female Genital Tuberculosis in a Tertiary Care Center- A Descriptive Cross-sectional Study.MRJI. 2018; 23(2): 1-6.

5. Bose M. Female genital tract tuberculosis: How long will it elude diagnosis? Indian $\mathrm{J}$ Med Res.2019;134(1):13-4.

6. World health organisation. Practical considerationsXpert MTB $\backslash R I F$ implementation manual, technical and operational 'How-to'. Geneva: WHO; 2014.

7. Blakemore R, Story E, Helb D, Kop J, Banada P, Owens MR, et al. Evaluation of the Analytical Performance of the Xpert MTB / RIF Assay. J Clin Microbiol. 2010; 48(7): 2495-501.

8. Helb D, Jones M, Story E, Boehme C, Wallace E, Ho K, et al. Rapid Detection of Mycobacterium tuberculosis and Rifampin Resistance by Use of
On-Demand , Near-Patient Technology. J Clin Microbiol. 2010; 48(1): 229-37.

9. Agarwal N, Gupta M, Garg R. Gene Xpert in diagnosis of female genital tuberculosis: an upcoming modality.Santosh University Journal of Health. 2017; 3: 48-9.

10. Kriplani A, Dharmendra S, Chaubey J, Kumar S, Sharma SK. Role of Gene Xpert in diagnosis of female genital tuberculosis: a preliminary report. Eur J Obstet Gynecol . 2016; 207: 237-8.

11. Saxena R, Shrinet K, Jain M. Comparative study of genital tuberculosis diagnosis in women with infertility. Int J Sci Res. 2017; 6(7): 817-9.

12. Sharma SK, Kohli M, Yadav R. Evaluation of Xpert MTB / RIF assay performance in diagnosing extrapulmonary tuberculosis among adults in a tertiary care centre in India. Eur Respir J. 2014; 44: 1087-1090.

13. Ssengooba W, Respeito D, Mambuque E, Blanco S, Bulo H, Mandomando I, et al. Do Xpert MTB / RIF Cycle Threshold Values Provide Information about Patient Delays for Tuberculosis Diagnosis? PLoS ONE. 2016; 302:1-10.

\footnotetext{
Bineeta Kashyap ${ }^{1}$, Dibyashree ${ }^{2}$, Rituparna Saha ${ }^{3}$, N. P. Singh ${ }^{4}$, Puneeta Hyanki ${ }^{5}$, Ashwani Khanna ${ }^{6}$

${ }^{1}$ Associate Professor, Department of Microbiology; ${ }^{2}$ Post Graduate Student, Department of Microbiology; ${ }^{3}$ Senior Resident, Department of Microbiology; ${ }^{4}$ Director Professor and Head, Department of Microbiology; ${ }^{5} \mathrm{CMO}$ I/C, DOTS center, University College of Medical Sciences and Guru Teg Bahadur Hospital, New Delhi; ${ }^{6}$ State TB Officer, RNTCP, New Delhi.
} 